Mots. Les langages du politique

73 | 2003

Les discours de la guerre

\title{
Discours médical et économie professionnelle
}

Medical discourse and professional economy

Discurso médico y economia profesional

Delphine HAIUN

\section{OpenEdition}

Journals

Édition électronique

URL : https://journals.openedition.org/mots/16772

DOI : $10.4000 /$ mots. 16772

ISSN : 1960-6001

Éditeur

ENS Éditions

\section{Édition imprimée}

Date de publication : 1 novembre 2003

Pagination : 143-158

ISBN : 2-84788-043-7

ISSN : 0243-6450

\section{Référence électronique}

Delphine HAIUN, « Discours médical et économie professionnelle», Mots. Les langages du politique [En ligne], 73 | 2003, mis en ligne le 13 octobre 2008, consulté le 22 avril 2022. URL : http:// journals.openedition.org/mots/16772 ; DOI : https://doi.org/10.4000/mots.16772 
Delphine HAIUN ${ }^{1}$

\section{Discours médical et économie professionnelle}

Cette étude voudrait mettre en lumière la logique économique de la pratique médicale française. Dès qu'on considère la médecine en tant que pratique discursive $^{2}$, on peut rassembler ceux qui l'exercent en un corps de pratique ; ce corps collectif, à travers son discours propre, met en œuvre un éthos médi$\mathrm{cal}^{3}$ qui se prête à l'analyse, comme voie d'accès pour décrire un fait socioculturel.

La médecine, telle qu'elle s'est organisée en Occident, est instituée en pratique professionnelle. Ce que nous voudrions dégager, c'est non pas le niveau national de l'économie médicale, niveau qui caractérise une aire politique, mais ce niveau d'organisation interne, qui caractérise un champ de pratique. Or les statuts professionnels propres dont jouit le corps médical en tant que corps de métier, anciennement dit «corporation», répondent à une norme sociale spécifique : c'est la norme dite déontologique. En France, l'Ordre des médecins se porte garant de cette norme professionnelle, qui est énoncée dans un texte institutionnel, le Code de déontologie médicale 4 .

Ce texte est en premier lieu d'ordre juridique, et se rattache donc à la norme républicaine française. En effet, après avoir été rédigé par le Conseil national de l'Ordre des médecins, il est soumis au Conseil d'État, puis publié au Journal officiel comme décret ministériel, et ce depuis 1947. Mais il peut aussi être analysé selon des données culturelles et symboliques : il possède une valeur initiatique puisqu'il est signé par tout médecin au moment de son inscription à l'Ordre (inscription qui fait office de licence d'exercice sur le territoire

1. Université de Tel-Aviv - Adresse personnelle : Y. L. Gordon 78/7, Tel-Aviv 69972, Israë1 - eladel@attglobal.net ; delphine@012.net.il

2. Nous plaçons notre étude dans la perspective philosophique de M. Foucault, qui intègre pratique, contexte socio-historique, et discours traversant l'un et l'autre, en un ensemble signifiant cohérent.

3. Nous entendrons le terme d'éthos au sens aristotélicien d'image que se forge une (ou plusieurs) personne(s) à travers son (leur) discours, délibérément ou non.

4. Désormais CDM. Édition source: 1995, Code de déontologie médicale, Paris, Direction des journaux officiels. Le texte intégral figure sur le site internet de l'Ordre national des médecins (http://www.conseil-national.medecin.fr). 
français) ; le $C D M$ peut ainsi être comparé à une profession de foi du corps médical français, rappelant le serment d'Hippocrate. C'est encore un texte à haute teneur philosophique, enraciné dans divers courants éthiques, notamment kantiens, qui reflète un mode de pensée continental et universaliste.

Nous analyserons la version de 1995 en vigueur à ce jour, comme discours institutionnel structurant la pratique médicale professionnelle française. Notre but sera de rendre compte de son économie professionnelle. Attirons l'attention sur trois concepts qu'on utilisera souvent, proches mais à distinguer : financier (lié à l'argent), économique (au sens des flux financiers qui traversent la pratique, mais aussi des rouages qui la font tourner), commercial (lié à une modalité spécifique d'exercice professionnel).

Dans un premier volet de l'étude, nous analyserons le principe théorique selon lequel le discours médical définit l'économie de la profession. Un second volet dégagera ensuite quelques corollaires de cette économie revendiquée.

\section{Le rapport topique ${ }^{5}$ de la médecine au commerce}

Le texte du $C D M$ est constitué de 112 articles, la plupart très courts. Ceuxci sont organisés en titres et sous-titres comme suit :

Titre I : Devoirs généraux des médecins

Titre II : Devoirs envers les patients

Titre III : Rapports des médecins entre eux et avec les membres des autres professions de santé

Titre IV : De l'exercice de la profession

$1^{\circ}$ - Règles communes à tous les modes d'exercice

$2^{\circ}$ - Exercice en clientèle privée

$3^{\circ}$ - Exercice salarié de la médecine

$4^{\circ}$ - Exercice de la médecine de contrôle

$5^{\circ}$ - Exercice de la médecine d'expertise

Titre V : Dispositions diverses

On peut individualiser dans l'ensemble du corpus l'émergence d'un thème qu'on dira "financier », lié à la notion d'argent. Ce thème se retrouve très fréquemment; un repérage systématique de ses apparitions montre qu'elles se présentent toujours en fin d'unité : dans la seconde moitié du titre I; à la fin du titre II ; et enfin diffusé le long du titre IV qui est l'avant-dernier (en particulier en $\mathrm{IV}-2^{\circ}$ et $\left.\mathrm{IV}-3^{\circ}\right)$. Cette place privilégiée du thème financier en position finale montre que globalement, il est placé en retrait des préoccupa-

5. Se reporter à la note 14 pour des précisions méthodologiques. 
tions mises en avant par le CDM. On peut d'emblée associer cet ordre d'apparition à un souci rhétorique d'apparence, lié à la mise en discours d'un éthos professionnel.

Malgré la haute fréquence de ce thème lié à l'argent, les lexèmes financier et économique ne présentent aucune occurrence dans le corpus, effacement qui confirme notre hypothèse rhétorique. Quant à commerce, il présente une occurrence d'ailleurs très singulière, ainsi que commercial. Ces deux occurrences sont regroupées dans l'art. 19.

\section{Le topos commercial de la médecine}

Attachons-nous à la formulation du premier alinéa de l'art. 196 : « La médecine ne doit pas être pratiquée comme un commerce ».

La proposition se trouve à la forme passive, où la médecine ne constitue que le sujet apparent du groupe verbal devoir pratiquer, et où l'absence de complément d'agent témoigne de l'absence de sujet réel de l'action de pratiquer. Ce qui est mis en avant est une entité abstraite, la médecine, entrant en relation avec une autre entité abstraite qu'est le commerce. Or la mise en relation de deux entités abstraites appelle en général une définition théorique, du sujet à l'aide du prédicat. Le lecteur est entrainé à interpréter cette phrase comme un énoncé définitionnel concernant la médecine en tant que pratique économique.

Or cette définition attendue des modalités économiques de la médecine est triplement biaisée : au lieu de définir la médecine, on ne formule que la forme selon laquelle elle ne doit pas apparaître.

- Premier biais : l'énoncé, composé d'une proposition unique, est d'ordre non pas descriptif, mais prescriptif ou normatif; il peut être vu comme texte injonctif ${ }^{7}$ ou comme acte de langage directif $^{8}$. Un tel acte de langage est accompli grâce à l'auxiliaire modal devoir ; ce n'est pas de la réalité médicale qu'il s'agit, mais de son devoir-être, ce qui n'étonne pas dans un texte de loi.

- Second biais : l'auxiliaire est à la forme négative, ce qui témoigne d'une injonction négative. Ce n'est pas d'un idéal positif qu'il s'agit, mais d'une interdiction.

6. Nous soulignons ci-dessous, et désormais dans toute citation, les termes pertinents de notre analyse.

7. Selon la typologie de E. Werlich, 1979, Typologie der Texte : Entwurf eines textlinguistischen Modells zur Grundlegung einer Textgrammatik, Heidelberg, Quelle und Meyer.

8. Selon la taxinomie de J. Searle, 1982, Les actes de langage. Essai de philosophie du langage, Paris, Hermann (trad. de 1969, Speech Acts, Cambridge, Cambridge University Press). 
- Troisième biais : si le thème de l'injonction est bien la pratique de la médecine, le rhème en est la manière commerciale; la négation porte sur le complément circonstanciel de manière qu'est l'expression comme un commerce. Ce que comme permet, c'est de nier la façon, la forme, la ressemblance, l'apparence, bref l'éthos médical. Il semble que la négation de l'identité entre médecine et commerce soit impossible à exiger, autrement cette tournure déconcertante aurait été évitée.

Ainsi, c'est l'éthos, désiré ou plutôt fui par la médecine, qui se joue dans cet alinéa, au lieu de sa définition économique. Ce procédé d'hétérogénéisation entre niveau définitionnel ou abstrait, et niveau technique ou concret, permet de statuer à un niveau de généralité qui s'apparente sans s'y identifier à l'essence même de la profession médicale 9 .

La suite de l'art. 19 est moins riche à l'analyse :

Sont interdits tous procédés directs ou indirects de publicité et notamment tout aménagement ou signalisation donnant aux locaux une apparence commerciale.

Ici aussi, la préoccupation des apparences domine, et cette fois, explicitement. L'isotopie commerciale ${ }^{10}$ qui lie ce second alinéa au premier n'est évidente qu'aux derniers mots de l'article. C'est au lecteur de restituer la proposition logique suivante, pour assurer la cohésion textuelle ${ }^{11}$ avec le premier alinéa et garantir la lisibilité de ''article $^{12}$; il comprendra : " dans le but d'éviter de donner une apparence commerciale aux locaux médicaux, on interdit la publicité par le présent article ».

L'article 19 peut finalement être ramené à trois propositions :

1. Les médecins n'endossent pas explicitement la responsabilité de la forme de leur pratique ${ }^{13}$ (le complément d'agent où ils auraient dû être men-

9. Nous ne préjugeons ici en rien de la motivation ni du degré d'intention des divers procédés linguistiques mobilisés, dont nous ne faisons que décrire les tenants et les aboutissants.

10. Le phénomène d'isotopie survient dès qu'un sème commun à deux propositions consécutives permet le passage cohérent de l'une à l'autre (un sème étant la plus petite entité de sens). Ce concept a été élaboré en particulier par A. J. Greimas, 1966, Sémantique structurale, Paris, Larousse ; et F. Rastier, 1987, Sémantique interprétative, Paris, PUF.

11. Voir les critères de textualité selon G. Genette, 1982, Palimpseste. La littérature au second degré, Paris, Le Seuil.

12. Voir aussi les maximes conversationnelles de H.-P. Grice, 1979, « Logique et conversation », Communications 30, Paris, Le Seuil (trad. de « Logic and conversation », dans P. Cole et J. L. Morgan (éd.), 1975, Syntax and Semantics, vol. 3, Speech Acts, Academic Press, Inc., p. 41-58); en particulier la maxime de relation.

13. En présupposant que les auteurs du $C D M$ représentent à la fois la médecine et les médecins. 
tionnés n'existe pas; la forme de la pratique médicale est sous-entendue comme inhérente à l'entité théorique qu'est la médecine).

2. Il est impossible d'affirmer si la médecine est un commerce ou ne l'est pas (bien qu'il semble que si elle ne l'était pas, ce fait serait clairement énoncé au premier alinéa).

3. La médecine ne veut pas ressembler à un commerce (et ce qui l'y ferait ressembler est strictement interdit au second alinéa).

On en déduit le topos suivant ${ }^{14}$, qui se décline le long de l'axe du rapport médecine/commerce :

- première forme topique : la médecine s'apparente à un commerce, axiologisée négativement puisque déniée par le $C D M$;

- seconde forme topique, renversée : la médecine ne s'apparente pas à un commerce, axiologisée positivement.

Ce topos fondamental structure, au même titre qu'un axiome, le discours déontologique médical. On le désignera désormais comme le topos commercial de la médecine. C'est donc en interdisant l'assimilation commerciale que la profession médicale se définit économiquement - il n'est cependant pertinent d'interdire que ce qui est susceptible d'être pratiqué.

\section{Analyse actancielle}

Afin de comparer les pratiques médicale et commerciale, représentons leur structure actancielle respective. Ce type d'analyse, forgé par A. J. Greimas ${ }^{15}$ dans un cadre structuraliste, porte sur des éléments sémiotiques et a pour vocation d'identifier les catégories universelles de sens impliquées dans une action donnée. Son souci de l'action permet de l'associer à une perspective pragmatique du langage. Nous saisirons ainsi le processus économique élémentaire qui fonde l'acte médical, puis l'acte commercial.

Du point de vue qui nous intéresse, la profession médicale peut être très généralement définie comme une certaine transaction économique, laquelle mobilise plusieurs catégories actancielles :

- l'action effectuée, ou quête : c'est l'acte médical ou acte de soin (aidé par des adjuvants, freiné par des opposants);

14. Rappelons qu'un topos est un élément liant le sens d'un énoncé ; au sens aristotélicien, il s'agit d'un lieu logique à structure enthymématique ou de syllogisme tronqué ; il prend un caractère plutôt thématique et cohésif dans les théorisations de F. Rastier (ouvr. cit.) ; ou plutôt argumentatif et implicite dans les théorisations d'O. Ducrot et J.-C. Anscombre, 1983, L'argumentation dans la langue, Liège, Mardaga.

15. Ouvr. cit. 
- un actant principal ou sujet de l'action, qui effectue cette quête : il s'agit du médecin ;

- l'objet recherché par cette quête : c'est la santé du patient (précisément, souvent le retour à une santé perdue $)^{16}$; cet objet transite au cours de l'action vers un destinataire, lequel s'incarne en la personne du patient. Notons d'emblée un point essentiel : du fait de son rôle d'objet de quête, la santé se voit investie d'une valeur, au sens d'une orientation axiologique positive ${ }^{17}$.

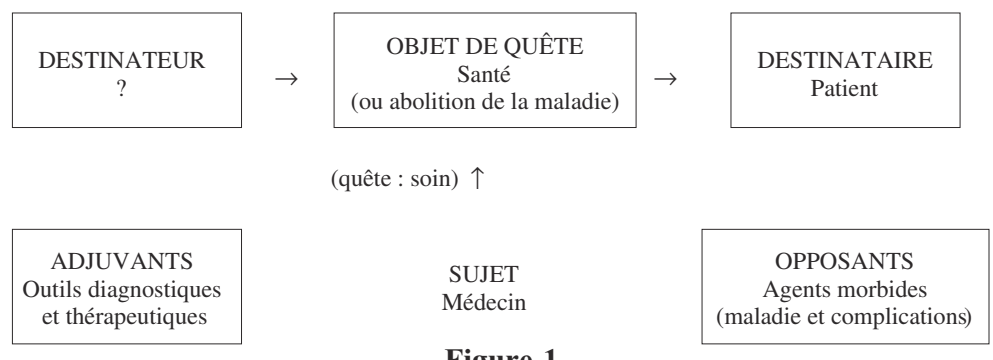

\section{Figure 1}

On peut dès lors re-spécifier l'acte médical : il constitue une transaction de la valeur-santé, effectuée par le médecin principalement ${ }^{18}$, pour le patient. La valeur qu'est la santé est doublement négociée : entre les adjuvants et les opposants; entre le médecin et son patient.

Dégageons maintenant la structure actancielle d'une transaction commerciale :

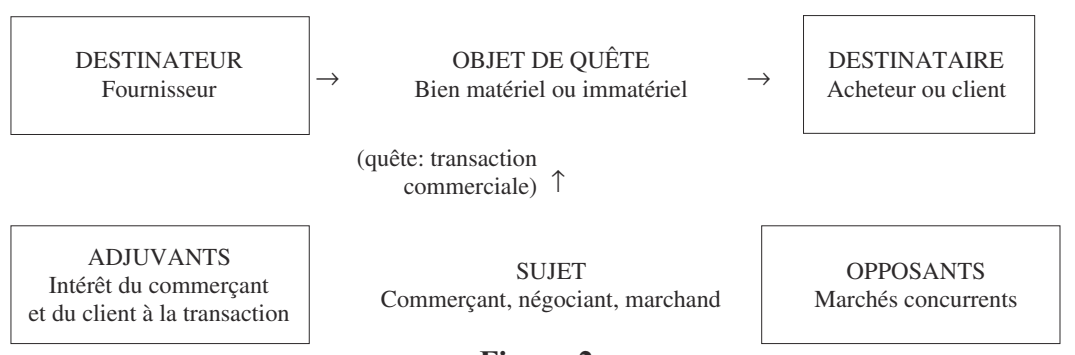

\section{Figure 2}

16. On ne discutera pas ici des distinctions entre buts thérapeutique, préventif et palliatif de la médecine, qui n'introduiraient que des nuances à l'analyse.

17. Voir C. Kerbrat-Orecchioni, 1980, L'énonciation. De la subjectivité dans le langage, Paris, Armand Colin.

18. Bien entendu l'action du médecin est menée conjointement à celle du patient, dont le rôle n'est pas que passif - mais ce fait, s'il a une signification éthique essentielle, est moins pertinent pour notre étude. 
La comparaison des deux schémas fait clairement ressortir leurs similitudes. La pratique médicale et la pratique commerciale peuvent toutes deux être vues comme des transactions rémunérées d'un certain objet de quête, institué d'une valeur du fait même de la quête. Dans le cas commercial, cette valeur est un bien matériel ou immatériel. Dans le cas médical, cette valeur est la santé.

Ainsi, la structure de la pratique médicale et celle de la pratique commerciale sont superposables. Qu'est-ce qui différencie alors ces deux pratiques?

\section{Analyse lexicologique}

Délimitons plus finement le sens communément admis pour chacun des lexèmes médecine et commerce. Nous nous aiderons du discours lexicographique, qui représente une synthèse du sens commun partagé par la communauté linguistique à laquelle il destine son usage, et est de ce fait bien adapté à une analyse topique ${ }^{19}$.

On a vu qu'il n'existe aucune définition explicite des modalités économiques de la profession médicale dans le corpus. Cherchons dans l'implicite.

Dès les premières lignes du $C D M$, l'article 2 affirme :

Le médecin, au service de l'individu et de la santé publique, exerce sa mission dans le respect de la vie humaine, de la personne et de sa dignité.

Certes, le lexème service est entendu ici dans l'acception suivante du Petit Robert: "Fait de se mettre à la disposition (de quelqu'un), par obligeance ». Il possède cependant d'autres sens qui colorent la médecine d'une nuance particulière, notamment en termes économiques.

De nombreuses acceptions de service contiennent un sens financier, parfois contradictoires: il suffit de comparer «ce que l'on fait pour quelqu'un ; avantage qu'on lui procure bénévolement»; et «ce qu'on fait pour quelqu'un contre paiement ou rémunération $»^{20}$.

19. Voir l'analyse du discours lexicographique préconisée par G.-E. Sarfati, 1995, Dire, agir, définir. Dictionnaires et langage ordinaire, Paris, L'Harmattan. On utilisera comme dictionnaire de référence A. Rey et J. Rey-Debove (dir.), 1991, Petit Robert. Dictionnaire de la langue française, Paris, Dictionnaires Le Robert. Outre qu'il constitue une autorité en langue française, ce dictionnaire se veut expressément structuré par le concept d'analogie, qu'on peut relier à celui d'isotopie et par là à celui de topos (voir la «Présentation du dictionnaire »); c'est dans cette perspective que nous utiliserons les associations proposées par le lexicographe.

20. Correspondant aux sens II, $2^{\circ}, 3^{\circ}, 4^{\circ}$ du Petit Robert. 
Relevons une acception du Petit Robert qui nous permettrait une définition :

(1875) Activités qui représentent une valeur économique sans correspondre à la production d'un bien matériel (surtout au pluriel; opposé à biens). Ex. : Société de services. Voir tertiaire (secteur).

Dans ce sens, la profession médicale s'assimilerait à un service du secteur tertiaire. Une précision essentielle est cependant ajoutée. Alors que l'analyse actancielle n'avait mis en évidence que la valeur axiologique, qualitative, prise par l'objet de quête du fait même qu'il était recherché, l'analyse lexicologique dégage une seconde valeur bien distincte, quantitative : la rémunération, le prix. Cependant un décalage saute aux yeux: si la pratique médicale attribue une valeur absolue à l'objet de quête - la santé -, l'économie médicale attribue une valeur relative et quantifiée à la quête elle-même - l'activité du médecin. C'est le principe du paiement à l'acte, typique des services, par opposition à un paiement au résultat. D'ailleurs, l'article $55 \mathrm{du}$ $C D M$ interdit aux médecins une rétribution forfaitaire garantissant au patient un retour à la santé ${ }^{21}$.

Reportons-nous maintenant à l'entrée commerce du Petit Robert. Le premier énoncé définitionnel (sens $\mathrm{I}, 1^{\circ}$ ) propose :

Opération qui a pour objet la vente d'une marchandise, d'une valeur, ou l'achat de celle-ci pour la revendre après l'avoir transformée ou non; entreprise qui fait cette opération.

Cet énoncé définitionnel met en scène la quête d'un objet par un sujet, rappelant la structure actancielle vue plus haut, et valide notre approche.

Deux termes complémentaires y sont utilisés : marchandise dans le sens de «bien matériel »; et valeur dont le caractère différentiel semble surgir de sa qualité immatérielle. En ce qui concerne la valeur financière (à bien distinguer de la valeur axiologique), c'est ici l'objet de transaction qui est vendu et/ou acheté, et a donc un prix, plutôt que la transaction elle-même.

Toujours sous le sens I, $1^{\circ}$ des relations lexicales vers libre-échange et protectionnisme nous rappellent les lois du commerce : par son principe de l'offre et de la demande, le commerce se rattache à l'idéal libéral ; mais,

21. Cette rétribution à l'acte semble d'ailleurs peu en faveur parmi les médecins (voir 2001, Pratiques - Les cahiers de la médecine utopique, $\mathrm{n}^{\circ} 13$ : La médecine et l'argent, Montpellier, Indigène); ceux-ci semblent cependant l'opposer à une rétribution horaire, qui permettrait d'éviter des consultations trop rapides, plutôt qu'à une rétribution forfaitaire liée au résultat, dont la possibilité ne semble jamais envisagée. C'est cette dernière opposition que nous examinons ici, à corréler à l'adage : «La médecine a obligation de moyen et pas de résultat $»$. 
d'autre part, il permet aussi, par l'imposition d'un contrôle interne, de protéger ou de préserver les commerçants. Les lois du commerce semblent presque s'élever en paradigme économique.

Le sens $I, 3^{\circ}$ propose :

Fonds de commerce. Voir : Boutique, débit, magasin. Exemples : Ouvrir, tenir un commerce. Gérance d'un commerce. Enseigne d'un commerce. Commerce à céder.

L'activité commerciale est située dans un lieu, rappelant l'ancrage de la pratique médicale dans le cabinet du médecin. De même, la notion d'enseigne, à lier à celle de publicité, souligne la signifiance de l'apparence extérieure. L'enseigne du médecin est assimilable à la plaque apposée devant le lieu d'exercice, dont l'utilisation est régulée de façon très détaillée par le $C D M$ :

Art. 81 : Les seules indications qu'un médecin est autorisé à faire figurer sur une plaque à son lieu d'exercice sont ses nom, prénoms, numéro de téléphone, jours et heures de consultations, situation vis-à-vis des organismes d'assurance-maladie, diplômes, titres et qualifications reconnus conformément au 4 et 5 de l'art. 79. Une plaque peut être apposée à l'entrée de l'immeuble et une autre à la porte du cabinet; lorsque la disposition des lieux l'impose, une signalisation intermédiaire peut être prévue. Ces indications doivent être présentées avec discrétion, conformément aux usages de la profession [...].

La discrétion imposée par le $C D M$ peut être rapportée au topos commercial de la médecine dégagé plus tôt.

Le sens I, $4^{\circ}$ de commerce est figuré : "Trafic de choses morales. Exemples : commerce honteux, infâme. Il fait commerce de son nom ». Ce sens, bien que n'étant pas qualifié par le Petit Robert de péjoratif, porte cependant cette nuance axiologique dans les termes mêmes utilisés par le lexicographe. D'ailleurs, à l'entrée commercial, s'individualise un sens déclaré péjoratif: "Conçu, exécuté dans une intention lucrative, et pour plaire au plus grand public ». L'axiologisation négative portée par la notion de commercial n'est évidemment pas souhaitée par le discours médical.

Quant au sens II de commerce, il est extrêmement élargi par rapport au sens I :

$1^{\circ}(1540)$ : Vieux ou littéraire. Relations que l'on entretient dans la société. V. Fréquentations, rapport; relation. Fuir le commerce des hommes. $2^{\circ}$ Manière de se comporter à l'égard d'autrui. V. Comportement, sociabilité. Locution moderne. Etre d'un commerce agréable.

Ce sens fait référence à la notion de relation interpersonnelle, contenue dans le signifié de commerce, et semble presque porter le schème commercial en parangon de la relation sociale. 
Une similitude structurelle, des différences économiques

La profession médicale semble donc préférer une définition économique en termes de service plutôt qu'en termes de commerce. Cette préférence peutelle être étayée ?

L'analyse actancielle a permis de dégager une structure pragmatique fondamentale, commune à plusieurs pratiques : la médecine constitue une transaction de la valeur que représente la santé, effectuée par le médecin et pour le patient - et il n'importe pas ici que la médecine soit considérée comme service ou comme commerce. Ainsi, l'opposition commerce/service ne s'avère pas signifiante d'un point de vue structurel; il s'agit en tout cas de la quête d'un objet doté de valeur, la santé.

Cependant, dans le cadre même de cette structure fondamentale de l'acte médical, ce qui est en jeu dans l'opposition commerce/service semble se centrer autour de deux points :

1. la chose payée : dans le commerce, c'est l'objet ou le produit final de la quête qui est payé ; dans un service, c'est la quête elle-même comme processus, action, activité, exercice, pratique ;

2. la matérialité de l'objet de quête : dans le commerce, la valeur recherchée peut être matérielle ou immatérielle ; dans un service, la valeur recherchée n'est pas matérielle.

Le premier point range clairement la médecine du côté des services puisque ce qui y est payé est l'acte médical. Dire que la médecine ne doit pas être pratiquée comme un commerce, c'est alors réaffirmer l'article 55 vu précédemment comme règle de paiement, et en corollaire, affirmer que la santé n'est pas achetée ${ }^{22}$. De là à déduire qu'elle n'est pas achetable, il n'y a qu'un pas, qui plonge cependant dans le domaine de la doxa, voire de l'idéologie du corps.

Assimiler la médecine à un service, plutôt qu'à un commerce, c'est effectuer une dichotomie entre la valeur recherchée (la santé), et la valeur payée (l'activité médicale). On assiste ici à la confrontation de deux systèmes normatifs entrant en concurrence sur un même terrain : le premier est d'ordre pragmatique (lié à l'intention et à la direction d'une action, d'une quête), le

22. Ce qui n'est pas lié à l'impossible garantie de résultat de la pratique médicale : il existe d'autres pratiques dont le résultat n'est que probable et/ou variable, et qui permettent cependant un paiement «au résultat». On peut concevoir un paiement proportionnel au résultat atteint, ou bien forfaitaire, auquel cas si aucune tentative n'aboutit des dédommagements peuvent être versés (citons en exemple les contrats industriels, ou parmi les services, les contrats immobiliers, où l'agent immobilier reçoit un pourcentage de la transaction effectuée). 
second est d'ordre économique (lié à l'institution d'un prix). Cette confrontation témoigne d'une incohérence de la médecine française voire occidentale, source potentielle de conflit: au lieu d'y payer directement ce qu'on veut obtenir, on paie indirectement un moyen de l'obtenir.

Le deuxième point concerne la matérialité de l'objet de quête, la nature ontologique attribuée à la santé. La santé a été définie par l'OMS ${ }^{23}$ comme « état de bien-être physique, psychologique et social », donc un état positif de l'être, plutôt qu'un avoir quelconque. Mais est-on en bonne santé ou bien at-on la santé : ne peut-on pas aussi penser la santé comme le bien le plus précieux dont on jouit? Valeur immatérielle, puisque qualité abstraite, ou bien matérielle, puisque incarnée dans un corps, voire possédée ? Postuler que la médecine ne doit pas être pratiquée comme un commerce de la santé, revient à préférer la valeur-santé comme qualité d'un être, plutôt que comme avoir quantitatif et monnayable.

On voit bien que les deux points se rejoignent. C'est toujours la santé qui est en jeu : la profession médicale semble répugner à en faire une valeur tangible, négociable, et, finalement, "saisissable par l'économique ».

\section{Champ sémantique de l'économie et du commerce}

Explorons maintenant quelques éléments du champ sémantique économique et commercial du $C D M$ qui corroborent notre analyse.

\section{Honoraires}

Le lexème le plus répandu pour désigner la rémunération de l'acte médical est celui d'honoraires (neuf occurrences, ce qui est peu relativement à la haute fréquence d'apparition du thème financier). Ce terme s'est formé d'après le latin honor, honoris, "témoignage de considération, charge, magistrature », donc valeur immatérielle. En dérive honorarium (donum), « don honorifique », donc valeur matérielle. Il désigne de nos jours la rétribution des professions libérales fournissant des services : une valeur non seulement matérielle, mais pécuniaire. Le rattachement du terme désignant la rémunération du médecin à une origine immatérielle et morale, non seulement confirme le rejet de la matérialité dans la pratique médicale, vu pour la notion de santé, mais encore, permet une axiologisation positive qui contribue à l'éthos prestigieux de la profession.

23. Organisation mondiale de la santé, organe satellite de l'Organisation des Nations unies. 
L'une des occurrences d'honoraires se situe à l'article 67, premier alinéa : «Sont interdites au médecin toutes pratiques tendant à abaisser, dans un but de concurrence, le montant de ses honoraires ». Le CDM ôte la pratique médicale du champ de la libre concurrence, opérant un protectionnisme. Cette logique de monopole est liée à une forte solidarité professionnelle qui se manifeste à de nombreuses reprises dans le corpus. L'article 56 est le plus probant : "Les médecins doivent entretenir entre eux des rapports de bonne confraternité. [...] Les médecins se doivent assistance dans l'adversité ».

\section{Client, clientèle}

L'analyse lexicométrique du corpus révèle l'absence du lexème client pour caractériser le patient ; un terme proche apparait néanmoins à quatre reprises, clientèle (une occurrence dans chaque titre, sauf dans le titre II).

Le $C D M$ s'intéresse principalement à la notion de clientèle en régulant la façon dont elle est susceptible de s'accroitre; l'article 57 postule : «Le détournement ou la tentative de détournement de clientèle est interdit». Cette interdiction est ultra-détaillée dans une dizaine d'articles, occupant presque tout le titre III.

Étymologiquement, un client est celui qui est protégé par une personne plus riche ou plus puissante que lui (du latin cliens, clientis), et déjà, à ce stade sémantique, donne clientèle comme «ensemble de protégés », puis, au $14^{\mathrm{e}}$ siècle, "celui qui a recourt aux services de quelqu'un ». Le sens économique n'apparait qu'au $19^{\mathrm{e}}$ siècle $^{24}$. Ainsi le topos assimilant le patient à un client, bien que rejeté par le discours déontologique contemporain, est justifié à la fois par l'étymologie et par l'usage moderne, allant même jusqu'à être admis par le discours lexicographique : le dictionnaire de médecine GarnierDelamare $^{25}$ définit directement le patient comme «client d'un médecin »; et à l'inverse le Petit Robert fournit patient comme premier ou second exemple des termes client et clientèle. Notons qu'il s'agit d'un changement d'approche, puisque les versions antérieures du CDM désignaient souvent le patient comme client $^{26}$. La notion de client caractérise tant la pratique commerciale

24. J. Picoche, 1983, Dictionnaire étymologique du français, Paris, Dictionnaires Le Robert.

25. J. Delamare, 1992, Le Garnier-Delamare. Dictionnaire des termes de médecine, $23^{\mathrm{e}}$ édition, Paris, Maloine.

26. Voir, par exemple, l'article 43 du Code de déontologie et statuts de la profession médicale de 1941: «Le secret professionnel lie le médecin d'une manière absolue ; il n'appartient pas au client de l'en délier »; cité par J. P. Alméras et H. Péquignot, 1996, La déontologie médicale, Paris, Litec. 
que les services; il semble que le discours médical y refoule simplement le caractère financier ou économique, et que ce phénomène soit récent.

Pour expliquer le contraste entre l'acceptation de clientèle et le rejet de client, on remarquera que le référent de client peut facilement être désigné par d'autres termes (parmi les plus usités dans le CDM: patient, 29 occurrences ; malade, 40 occurrences ; personne, 29 occurrences). En revanche, clientèle n'a aucun synonyme et son utilisation s'avère presque inévitable ; notons cependant l'apparition récente dans le discours médical d'un nouveau lexème, patientèle, qui souligne trop bien ce qu'il voudrait masquer : le rejet de l'aspect économique, associé à la fidélisation d'un groupe de protégés.

Le terme clientèle appelle un certain critère de groupage. Le droit d'exploitation présupposé par le $C D M$ est en effet fondé sur un critère géographique ; or ce droit participe du monopole d'activité évoqué plus haut. L'attribution d'une "zone d'action potentielle » à chaque médecin élargit singulièrement le concept de clientèle, d'un état de fait à un état de droit. En toute rigueur sémantique, le concept de clientèle pose les limites du concept de libre concurrence. C'est clairement la vision clientéliste qui domine la vision concurrentielle dans la médecine française - ce qui va dans le sens du protectionnisme vu plus haut - et la médecine pour se défendre utilise classiquement un argument certes valable, mais typiquement commercial : « fidéliser c'est pour mieux servir $»^{27}$.

La négociation des termes client, clientèle et patient semble témoigner d'un enjeu serré où la médecine est dépouillée d'un caractère de libre échange économique. En même temps, ce sont véritablement les droits valorisant la personne soignée qui sont détournés d'elle : si les clients sont dotés de droits par la doxa (le client a toujours raison), sans parler de la loi, le terme patient se forme sur un étymon pour le moins dévalorisant - pati, passus, « souffrir, supporter, patienter passivement ${ }^{28}$, non seulement la maladie, mais encore la pratique médicale.

\section{Éthos et publicité}

Éthos et publicité sont deux thèmes qui se retrouvent souvent dans les mêmes articles ou tout au moins dans les mêmes zones du $C D M$, aussi en déduit-on qu'ils sont en relation d'isotopie et ont au moins un sème en com-

27. Voir les commentaires du CDM rédigé par l'Ordre des médecins en vue de l'expliquer aux médecins intéressés.

28. J. Picoche, ouvr. cit. 
mun. Si l'éthos est l'apparence, l'image d'un sujet telle qu'il la forge, la publicité constitue la mobilisation de cette image dans un but de rentabilité financière : la publicité est le versant économique de l'éthos.

Le rapport du $C D M$ envers la publicité est nettement évitant, comme le formule l'article 19 longuement analysé. Aux articles 79 à 82 , sont minutieusement régulées les indications mentionnées sur la feuille d'ordonnance, la plaque, l'annuaire, ou une annonce dans la presse. Il est interdit au médecin d'user de son influence en faveur de causes commerciales (art. 13, 20, 26, 27). Ce qui est explicitement contrôlé, ce sont les mentions du nom et de la qualité du médecin, comme si ces mots incarnaient le prestige de la profession.

Examinons l'entrée publicité du Petit Robert. Le sens 1 ne concerne qu'une mise à disposition du public, mais le sens 2 s'oriente vers un caractère précisément commercial ( «le fait, l'art d'exercer une action psychologique sur le public à des fins commerciales »), et se voit en outre mis en rapport lexématique avec annoncer, propagande, image de marque, texte à caractère publicitaire, autant de notions liées à celle d'éthos. Relevons à ce propos la formulation de l'article $82:$ «[...] le médecin peut faire paraitre dans la presse une annonce sans caractère publicitaire [...] ». Or une annonce dans la presse est par définition la mise à disposition publique d'une certaine information, et constitue en ce sens une publicité au sens premier, sans parler du fait qu'elle est bien destinée à constituer une clientèle ; le $C D M$ n'en renie que le caractère économique.

L'éthos de la profession médicale se démarque ainsi fortement d'un éthos économique, mais est-il constitué de traits positifs ? La silhouette du médecin qui se dessine au fil des articles, et qui concerne plus l'homme que le professionnel, le veut respectable, prudent, subtil, loyal, réconfortant, solidaire, conciliant, objectif, etc., autant de qualités qui sont liées à un désintéressement. L'article 31, dernier du titre I, concerne la préservation de cet éthos : «Tout médecin doit s'abstenir, même en dehors de l'exercice de sa profession, de tout acte de nature à déconsidérer celle-ci. » Cet article fait preuve d'un protectionnisme très prononcé : par souci de ne pas entacher l'éthos professionnel, le corps médical va jusqu'à empêcher les médecins de manifester une opposition publique, justifiée ou non, rejoignant l'article 56 (« les médecins se doivent assistance dans l'adversité »). Dès qu'on tient le public pour tiers garant d'une certaine clairvoyance, autant censurer une autocritique éclairée. La confraternité est poussée jusqu'à retirer à la communauté des patients son rôle d'arbitre, le réservant à l'Ordre.

Rassemblons nos résultats. La pratique médicale possède une structure commerciale, mais un objectif immatériel, la santé, assimilable à une valeur 
pragmatique puisque recherchée par l'acte médical, mais qui se proclame insaisissable par l'économique. «La santé n'a pas de prix » : l'adage dit bien que sa valeur est infinie, pour ne pas dire sacrée ; c'est une position dogmatique qui se profile ici. L'éthos médical, désinvesti de toute apparence financière et valorisant l'acte en le dotant exclusivement d'un prix, y gagne en prestige moral ; mais le patient y perd ses droits dans la transaction médicale, premièrement de client, et secondement de juge. La profession médicale fonctionne ainsi en monopole fermé, où clientélisme et protectionnisme prévalent ; cette forte confraternité professionnelle aboutit à une prise de pouvoir du corps médical sur le corps du patient.

Un professionnel est défini comme «personne physique ou morale qui exerce à titre habituel une activité économique réglementée $»^{29}$. Or les modalités économiques de la profession médicale, réduites à une allusion indirecte à la notion de service, refusent d'être explicitement définis par le $C D M$, revendiquant une caractérisation propre et originale qui semble relever de l'indicible, voire de l'absolu. D'ailleurs un président du Conseil National de l'Ordre des médecins a proclamé :

S'appuyant sur une solide tradition enracinée dans l'Antiquité, l'exercice de la médecine est soumis à des lois qui engagent tous ceux qui ont accepté de remplir cette exceptionnelle mission. Cette continuité dans le respect de règles universelles et éternelles, constitue l'essence même de la déontologie ${ }^{30}$.

Nous ne soulignerons plus le désintéressement qu'implique le terme de mission. Ce qu'il convient de relever pour conclure, c'est la contradiction sous-tendant ces lignes, qui trahit une certaine illusion : la nature originale et particulière de la pratique médicale, dite exceptionnelle, est loin d'être universelle et encore moins éternelle, mais nous semble plutôt relever de l'institution culturelle d'une pratique en profession, pétrie d'idéologie et d'histoire, depuis le serment d'Hippocrate jusqu'aux lois de la République, dont les détails restent à analyser.

$\mathrm{Au}$ terme de cette étude portant sur un corpus français, on peut émettre l'hypothèse que certaines de ses conclusions s'étendent à l'ensemble de la pratique médicale continentale, voire occidentale. Quelques catégories mises en jeu, comme celle de profession (en tant que pratique discursive), de patient, de client, s'ancrent dans les fondements même de notre civilisation. Quant à la santé, elle s'avère prendre son sens en contexte puisqu'elle est

29. J.-L. Sourioux \& P. Lerat, 1992, L'analyse de texte. Méthode générale et applications au droit, Paris, Dalloz-Sirey.

30. Préface de B. Glorion, dans B. Hoerni, 1996, Éthique et déontologie médicale, Paris, Masson. 
chargée de valeur par l'acte médical : une valeur non seulement sémiotique, dans le cadre d'une pragmatique linguistique, mais aussi historique et culturelle ${ }^{31}$.

\section{Résumé / Abstract / Compendio}

Discours médical et économie professionnelle

La médecine française s'organise en pratique professionnelle régie par le Code de déontologie médicale, texte normatif à visée éthique, mobilisant des valeurs juridiques, philosophiques et symboliques. Une analyse discursive de la version en vigueur montre que les modalités économiques de la profession y sont refoulées. Pourquoi ce désinvestissement ? Quels signifiés contextuels et culturels sont mis en cause ?

Mots clés : Analyse du discours, linguistique pragmatique, pratique discursive, économie médicale, déontologie professionnelle.

\section{Medical discourse and professional economy}

French medicine is organized as a professional practice, ruled by the Code de déontologie médicale, a normative text using legal, philosophical and symbolic values to express ethical norm. A discursive analysis of its up-to-date version shows that the economical aspect of the profession is driven back. Why? Which semantic, contextual, and cultural categories of sense are involved in this process?

Key words : Discourse analysis, pragmatic linguistics, discursive practice, medical economy, professional ethics.

\section{Discurso médico y economia profesional}

La medicina francesa esta organizada como una practica profesional regida por el Código de deontología médica, un texto jurídico con un alto contenido filosófico y simbólico. Un análisis discursivo de la versión vigente evidencia que las modalidades económicas de la profesión se hallan refundidas ¿Por qué? ¿Qué significantes contextuales y culturales están involucrados en este proceso?

Palabras claves : Análisis del discurso, pragmatismo lingüistico, práctica discursiva, economía médica, deontología profesional.

31. Pour tout développement, voir D. Haiun, 2002, Analyse d'un discours institutionnel : le Code de déontologie médicale français de 1995 - Topiques politique et économique, enjeux éthiques, thèse déposée à l'université de Tel-Aviv, se proposant pour publication. 